\title{
Study of V/Q Non-Linearity in Scientific CMOS Sensor
}

\author{
Jun-Lin $\mathrm{Li}^{1,2, *}$, Yong-Fei Guo ${ }^{1}$, Tai-Ji Lan ${ }^{1,2}$ \\ ${ }^{1}$ Changchun Institute of Optics, Fine Mechanics and Physics, Chinese Academy of \\ Sciences, Changchun, Jilin 130033, China \\ ${ }^{2}$ University of Chinese Academy of Sciences, Beijing 100049, China \\ lijunlin0913@126.com,guoyf@ciomp.ac.cn,lantaiji@tju.edu.cn
}

\begin{abstract}
Scientific CMOS sensor usually manifests $V / Q$ non-linearity in charge-to-voltage conversion. Starting from the mechanism underlying this non-linearity, we build the $V / Q$ non-linearity model to study the influence of modulation transfer function (MTF) and signal-to-noise ratio (SNR). Meanwhile, simulation verification is carried out. The results show that V/Q non-linearity improves SNR but causes the decrease of MTF of the electronic device. We propose the combination of video response curve with photo response non-uniformity (PRNU) noise curve to locate V/Q non-linearity. The validity of this method is proved by simulation verification and physical experiment. The present study provides reference for design optimization and compensation for non-linearity in scientific CMOS sensor.
\end{abstract}

Keywords: CMOS, V/Q non-linearity, modulation transfer function (MTF), signal-tonoise ratio $(S N R)$

\section{Introduction}

The performance of scientific CMOS sensors is gradually approaching that of scientific CCD sensors due to the advances in CMOS technique. Currently CMOS sensors have found wide applications in deep space exploration and space remote sensing. Response non-linearity is among the core indicators for assessing the performance of an image sensor $^{[1,2]}$. While different types of non-linearity are produced during photoelectric conversion ${ }^{[3]}$, the present study focuses on V/Q non-linearity specific to CMOS sensor.

After light signals reach the light-sensitive surface of CMOS sensor, they are converted into digital signals through photo-electric conversion, charge measurement, signal sampling and $\mathrm{AD}$ conversion. Three conversions take place in succession, namely, photon to electron, electron to voltage, and voltage to code value ${ }^{[4,5]}$, and all of them are supposed to be linear under ideal situations. In reality, non-linearity occurs after all, and non-linearity in electron to voltage conversion is defined as V/Q non-linearity; the nonlinearity in voltage to code value conversion is defined as DN/V non-linearity. For CCD sensors, DV/V non-linearity is paramount, while for CMOS sensors, V/Q non-linearity is paramount $^{[6,7]}$.

Modulation transfer function (MTF) and signal-to-noise ratio (SNR) are the core performance indicators of scientific image sensors. They reflect the discrimination capacity of the sensors for objects with different spatial resolution ${ }^{[8,9]}$ and under low irradiance. We emphatically analyze the influence of V/Q non-linearity on MTF and SNR. ${ }^{1}$

Response non-linearity of CMOS sensor is more intuitively manifested on the video response curve, but it is difficult to determine which type of non-linearity is the leading one. We propose the method of combining video response curve with photo response non-

Jun-Lin Li is the corresponding author. 
uniformity (PRNU) noise curve to locate V/Q non-linearity. Based on theoretical derivation, we perform simulation analysis and experimental verification, which demonstrate the validity of the proposed method.

\section{V/Q Non-Linearity}

V/Q non-linearity in CMOS sensor is the non-linearity arising from electron to voltage conversion. The equivalent model of the conversion under ideal conditions is shown in formula (1). The measured capacitance $\mathrm{C}$ is a constant during the integral process. In a real CMOS sensor, the charge measurement unit is mainly in the form of a photodiode, which works under reverse bias voltage and undergoes the integral process ${ }^{[10-12]}$. The equivalent circuit of the photodiode is shown in Figure 1, where the equivalent capacitance $\mathrm{C}_{\mathrm{d}}$ of diode is the capacitance measured based on charge. According to physical theory of semiconductor devices, the physical equivalent model of $\mathrm{C}_{\mathrm{d}}$ is shown as formula (2), where A is area of PN node and M is the dielectric coefficient, a parameter related to the doping concentration of semiconductor material. For a given CMOS sensor, both $\mathrm{A}$ and $\mathrm{M}$ are constant, and $\mathrm{V}$ is the on-load voltage on the two sides of equivalent capacitance. Thus, $\mathrm{C}_{\mathrm{d}}$ the capacitance measured based on charge is not a constant during charge measurement, but is inversely proportional to the $1 / 2$ exponent of voltage $\mathrm{V}$. The proportionality coefficient is directly related to A and $\mathrm{M}$, which lead to V/Q non-linearity.

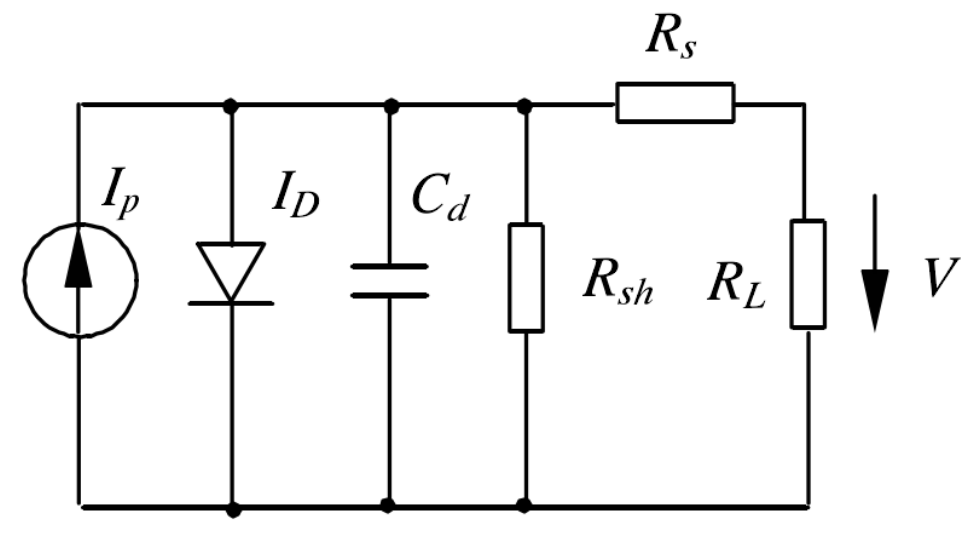

Figure 1. Equivalent Circuit of the Photodiode

$$
\begin{aligned}
& Q_{P I X}=\int_{V_{T E}}^{V_{R E F}} C d V=C \times\left(V_{R E F}-V_{T E}\right)=C \times V_{P I X} \Rightarrow V_{P I X}=\frac{Q_{P I X}}{C} \\
& C_{d}=A M V^{\frac{1}{2}}
\end{aligned}
$$

Substituting (2) into (1) for the integral process, the model of V/Q non-linearity is obtained as formula (3), where $\mathrm{V}_{0}$ is the voltage reference after reset. The output voltage $\mathrm{V}_{\text {PIX }}$ is no longer linearly related to the measured amount of charge $\mathrm{Q}_{\text {PIX }}$, but through a quadratic function. V/Q non-linearity can be expressed as formula (4), and the nonlinearity increases with the increasing amount of charge (incident irradiance), peaking at full well capacity. Generally, $\mathrm{Q}_{\mathrm{P}}$ is far smaller than $2 \mathrm{AMV}_{0}{ }^{1 / 2}$ (the ratio between the two is smaller than 5\%), and therefore V/Q non-linearity is negligible. However, for the reason of manufacturing process or of ensuring the sensitivity of CMOS sensor, $2 \mathrm{AMV}_{0}{ }^{1 / 2}$ is smaller than this. Then the ratio of $\mathrm{Q}_{\mathrm{P}}$ to $2 \mathrm{AMV}_{0}{ }^{1 / 2}$ will exceed $5 \%$ or even $20 \%$. At this time, V/Q non-linearity has a non-negligible impact on the imaging system. Figure 2 is the V/Q non-linearity represented in a rectangular coordinate system, where $\mathrm{X}$ axis is the charge amount $\mathrm{Q}_{\mathrm{PIX}}$, and $\mathrm{Y}$ axis is the voltage $\mathrm{V}_{\mathrm{PIX}}$.

$$
V_{P I X}=\frac{\frac{Q_{p}}{A M}\left(2 V_{0}^{\frac{1}{2}}-\frac{Q_{p}}{A M}\right)}{\left[\frac{\mathcal{C}_{p}}{A M} 2 V_{0}^{\frac{1}{2}}-\frac{\mathcal{Q}_{p}^{A M}}{A M}\left(2 V_{0}^{\frac{1}{2}}-\frac{Q_{p}}{A M}\right)\right]} \times 100 \%=\frac{Q_{p}}{2 A M V_{0}^{\frac{1}{2}}} \times 100 \%
$$




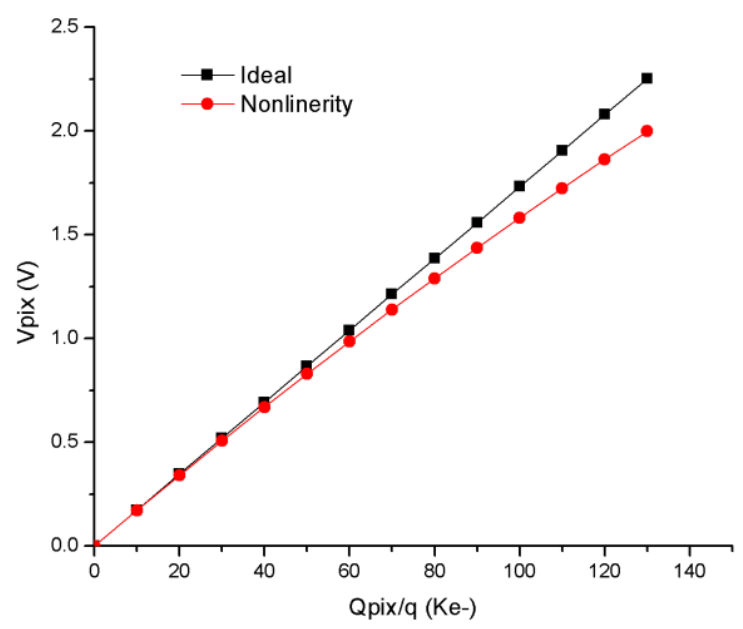

Figure 2. V/Q Non-Linearity in Simulation

\section{Influence of V/Q Non-Linearity on Photoelectric Parameters}

\subsection{Influence of V/Q Non-linearity on SNR}

According to noise distribution model of the CMOS sensor, the largest source of noise is the photon shot noise. Here SNR is modeled based on the shot noise. SNR transfer under ideal conditions is expressed as formula (5), and SNR does not change.

$S N R_{D N}=\frac{S_{D N}}{N_{D N}}=\frac{Q_{P} \times G / C}{\sqrt{q Q_{P}} \times G / C}=\sqrt{\frac{Q_{P}}{q}}=S N R_{Q}$

However, in the presence of V/Q non-linearity, the relation between $\mathrm{SNR}_{\mathrm{DN}}$ and $\mathrm{SNR}_{\mathrm{Q}}$ can be derived as (6) from (3) and (5). Due to V/Q non-linearity, the conversion gain of the signals is larger than the conversion gain of noise. This has the equivalent effect that the noise is suppressed and therefore SNR is improved. The curve showing the variation of SNR with irradiance is shown in Figure 3. However, the difference in conversion gain of signals and noise will lead to the change of the gain of the imaging system, which is unacceptable to the applications in surveying and mapping and spectral analysis.

$S N R_{D N}=\frac{\frac{Q_{P}}{A M}\left(2 V_{0}^{\frac{1}{2}}-\frac{Q_{P}}{A M}\right)}{\frac{\sqrt{q Q_{P}}}{A M}\left(V_{0}-V_{P I X}\right)^{\frac{1}{2}}}=\sqrt{\frac{Q_{P}}{q}} \times\left(\frac{2 V_{0}^{\frac{1}{2}}-\frac{Q_{P}}{A M}}{V_{0}^{\frac{1}{2}}-\frac{Q_{P}}{A M}}\right)>S N R_{Q}$ 


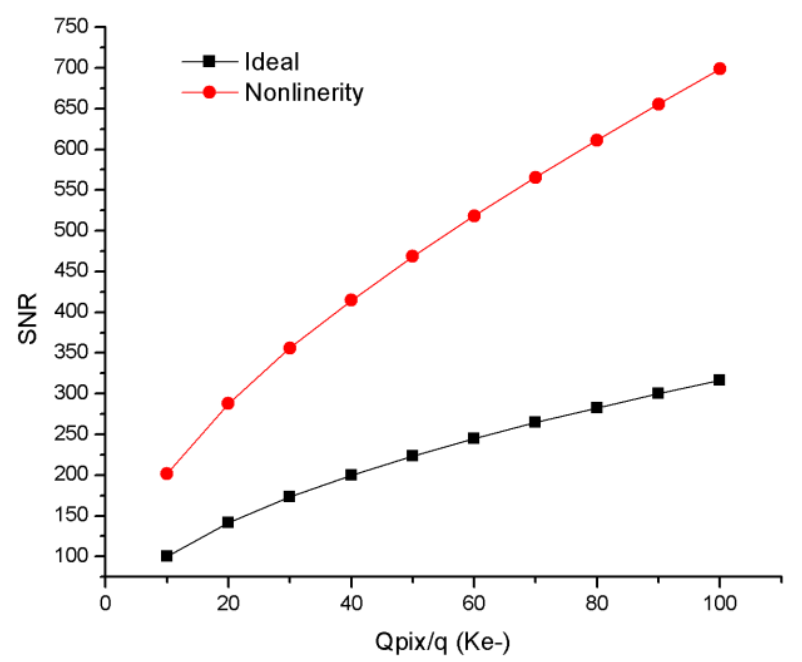

Figure 3. Variation of SNR with Irradiance

\subsection{Influence of V/Q Non-Linearity on MTF}

MTF is defined as the ratio of output modulation to input modulation. Ideally, MTF is only the function of spatial frequency $\omega$. MTF curve in the CMOS sensor is represented by formula (7), where $\mathrm{G}$ is the gain of voltage to code value conversion and $\mathrm{C}$ is the gain of charge to voltage conversion. No loss occurs during conversion.

$M T F_{Q}=\frac{Q_{\text {max }}-Q_{\text {min }}}{Q_{\text {max }}+Q_{\text {min }}}=\frac{\left(Q_{\text {max }}-Q_{\text {min }}\right) \times G / C}{\left(Q_{\text {max }}+Q_{\text {min }}\right) \times G / C}=\frac{D N_{\text {max }}-D N_{\text {min }}}{D N_{\text {max }}+D N_{\text {min }}}=M T F_{D N}$

The relation between $\mathrm{MTF}_{\mathrm{DN}}$ and $\mathrm{MTF}_{\mathrm{Q}}$ will change into formula (8) in the presence of $\mathrm{V} / \mathrm{Q}$ non-linearity. $\mathrm{MTF}_{\mathrm{DN}}$ is not only the function of spatial frequency $\omega$, but also changes with $\mathrm{Q}_{\max }$. Irradiance being constant, the curve showing the variation of $\mathrm{MTF}_{\mathrm{DN}}$ and $\mathrm{MTF}_{\mathrm{Q}}$ with the spatial frequency is plotted in Figure4. Thus $\mathrm{MTF}_{\mathrm{DN}}$ is below the ideal value at any frequency point, which means that there is a loss due to V/Q non-linearity. Under Nyquist frequency, the variation of $\mathrm{TF}_{\mathrm{DN}}$ and $\mathrm{MTF}_{\mathrm{Q}}$ with irradiance can be represented by the curve in Figure5. It can be seen that V/Q non-linearity is the reason for the changing of MTF with irradiance; the higher the irradiance, the lower the MTF.

$M T F_{D \mathrm{~N}}=\frac{\frac{Q_{\max }}{A M}\left(2 V_{0}^{\frac{1}{2}}-\frac{Q_{\max }}{A M}\right)-\frac{Q_{\min }}{A M}\left(2 V_{0}^{\frac{1}{2}}-\frac{Q_{\min }}{A M}\right)}{\frac{Q_{\max }}{A M}\left(2 V_{0}^{\frac{1}{2}}-\frac{Q_{\max }}{A M}\right)+\frac{Q_{\min }}{A M}\left(2 V_{0}^{\frac{1}{2}}-\frac{Q_{\min }}{A M}\right)}=\frac{\left(Q_{\max }-Q_{\min }\right)\left[2 V_{0}^{\frac{1}{2}}-\frac{\left(Q_{\max }+Q_{\min }\right)}{A M}\right]}{\left(Q_{\max }-Q_{\min }\right)\left[2 V_{0}^{\frac{1}{2}}-\frac{\left(Q_{\max }+Q_{\min }\right)}{A M}\right]+\frac{2 Q_{\max } Q_{\min }}{A M}}<M T F_{Q}$ 


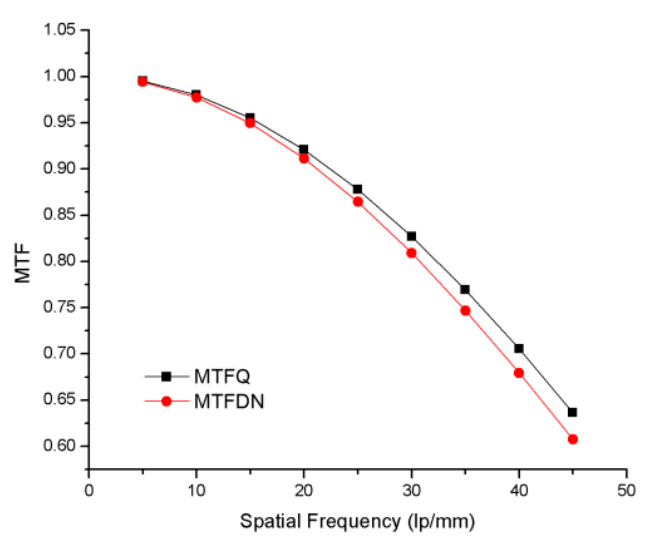

Figure 4. Variation of MTF with Spatial Frequency

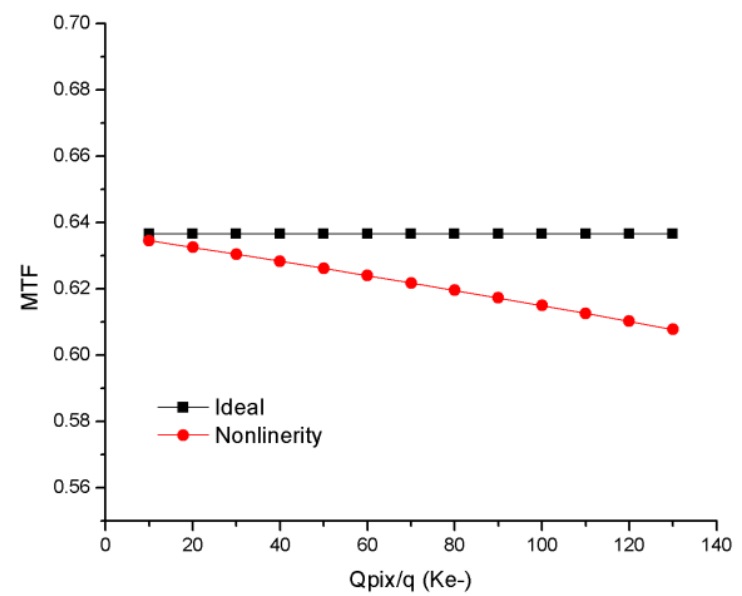

Figure 5. Variation of MTF with Irradiance

\section{Method for Judging V/Q Non-Linearity}

Non-linearity of the CMOS sensor can be more clearly visualized by plotting the video response curve. However, the video response curve alone cannot differentiate V/Q nonlinearity from other types of non-linearity. Therefore we propose the method of locating V/Q non-linearity by combining the video response curve with PRNU noise curve.

\subsection{Plotting of PRNU Noise Curve and Testing}

For single pixels of the CMOS sensor, the response within the dynamic range is considered a constant ${ }^{[13,14]}$. However, the response of different pixels varies, and this difference is defined as PRNU noise, measured by noise factor $\Phi$. Formula (9) is the mathematical model of $\Phi$ under ideal conditions. $\mathrm{DN}_{\mathrm{Av}}$ and $\sigma$ are mean and standard deviation of DN of all pixels after removing photon shot noise and black level correction, respectively; $n$ is the number of pixels. The variation curve of noise factor $\Phi$ with irradiance is the PRNU noise curve, which is supposed to a horizontal straight line under ideal conditions.

$$
\Phi=\frac{\sigma}{D N_{A V}}=\frac{n}{n-1} \times \frac{\sqrt{\sum_{i=1}^{n}\left(R_{i}-\frac{1}{n} \sum_{i=1}^{n} R_{i}\right)^{2}}}{\sum_{i=1}^{n} R_{i}}
$$


To test the PRNU noise curve, dark frame test is first carried out. With the obtaining of $\mathrm{N}$ (1000) dark frame images, the mean $\mathrm{DN}_{\mathrm{di}}$ and mean square deviation $\sigma_{\mathrm{DTOi}}{ }^{2}$ are calculated for each image. Then the means of $\mathrm{DN}_{\mathrm{di}}$ and $\sigma_{\mathrm{DTOi}}{ }^{2}$ are calculated to obtain the dark signal $\mathrm{DN}_{\mathrm{da}(\mathrm{i}, \mathrm{j})}$ and the noise of dark signal $\sigma_{\text {Dто. }}$

In the subsequent bright frame test, the step length is increased by 1 for the output irradiance of the integrating sphere, and $\mathrm{N}$ frames of images are collected after the light source is stabilized. The mean $\mathrm{DN}_{\mathrm{P}}(\mathrm{i}, \mathrm{j})$ of $\mathrm{N}$ samplings for each pixel under the corresponding irradiance is calculated, and these values form a new image. Then by calculating the mean $\mathrm{DN}\left({ }_{\mathrm{i}, \mathrm{j}}\right)$ and the standard deviation $\sigma_{\mathrm{TO}}$ for this image, the dark noise is subtracted, thus obtaining the noise factor $\sigma_{\mathrm{TO}}$. The output irradiance is increased until the device under the test achieves saturated output. The above procedures are repeated to obtain the sequence of noise factors $\{\Phi \mathrm{i}\}$.

Next PRNU noise curve is plotted with output irradiance on the $\mathrm{X}$ axis and $\Phi$ on $\mathrm{Y}$ axis, and the test is over.

\subsection{Method for Judging V/Q Non-Linearity}

In the presence of DN/V non-linearity or other types of non-linearity, the video response will be non-linear, but the PRNU noise curve is still a horizontal straight line.

When there is V/Q non-linearity, the slope $\Phi$ of PRNU curve is calculated by formula (10) by combining (3) and (9).

$$
\phi=\frac{n}{n-1} \cdot \frac{\sqrt{\sum_{i=1}^{n}\left[Q_{i}\left(2 V_{0}^{\frac{1}{2}}-\frac{Q_{i}}{A M}\right)-\frac{\sum_{i=1}^{n} Q_{i}\left(2 V_{0}^{\frac{1}{2}}-\frac{Q_{i}}{A M}\right)}{n}\right]^{2}}}{\sum_{i=1}^{n} Q_{i}\left(2 V_{0}^{\frac{1}{2}}-\frac{Q_{i}}{A M}\right)}
$$

With integral time fixed, the results of simulation of video response curve and PRNU curve under the changing incident irradiance are shown in Figure 6 and 7, respectively. It can be seen that the video response curve is non-linear, while PRNU noise curve is also non-linear and decreases with the increasing irradiance.

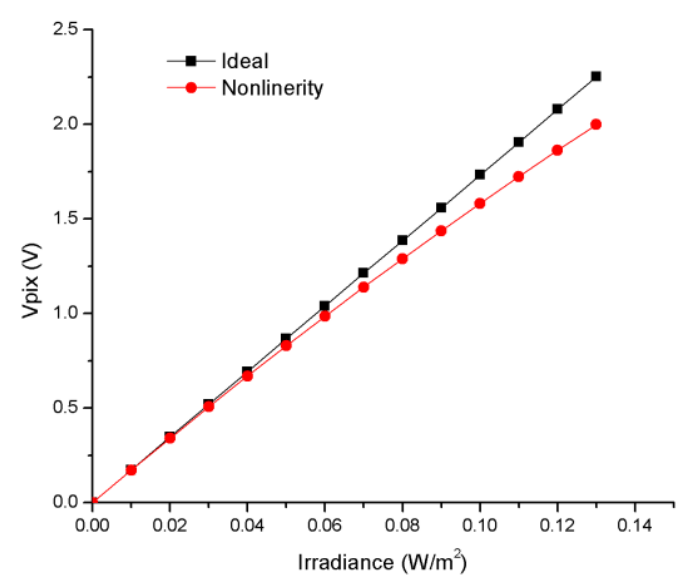

Figure 6. Video Response Curve 


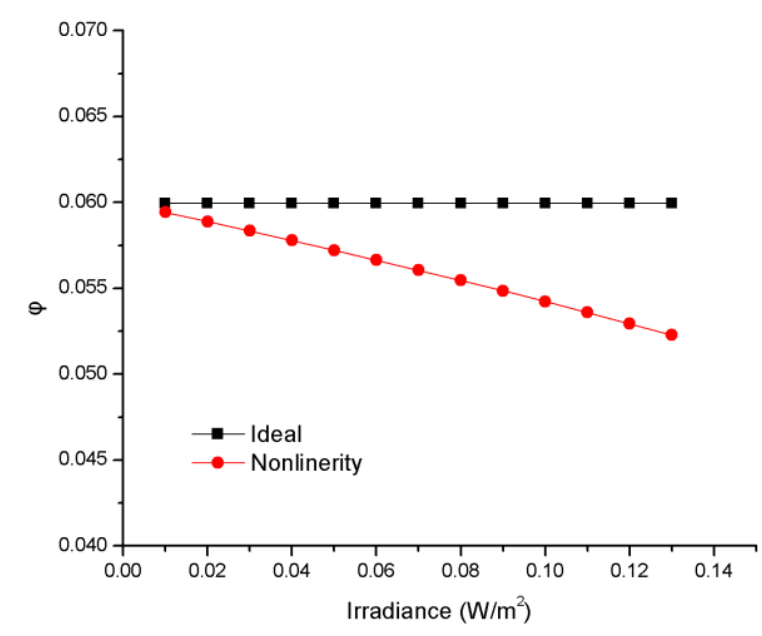

Figure 7. PRNU Noise Curve

V/Q non-linearity increases with the increase of measured amount of charge in CMOS sensor. Therefore, for CMOS sensors having maximum non-linearity not above $10 \%$, the high-response interval for 5\%-10\% non-linearity can be corrected by look-up table method. Then the correction effect can be verified by the testing of PRNU curve. For CMOS sensors with maximum non-linearity above $10 \%$, replacement or other treatments should be performed.

\section{Experimental Verification}

The proposed method for judging V/Q non-linearity in CMOS sensor is verified experimentally. The experiment environment is displayed in Figure 8. Halogen lamp is used as the integrating sphere, and the diaphragm is adjusted with a motor to ensure an adjustable irradiance under fixed color temperature. Closed-loop automation control of the irradiance is realized by using a standard illuminometer. Irradiance output at equal step length from dark frame to saturation using the integrating sphere, and shooting and image collection and storage with CMOS sensor are all placed under computer-assisted control. Video response curve and PRNU noise curve are plotted after processing of the raw image data. Finally, the presence of V/Q non-linearity is judged according to the variation trend of the curves.

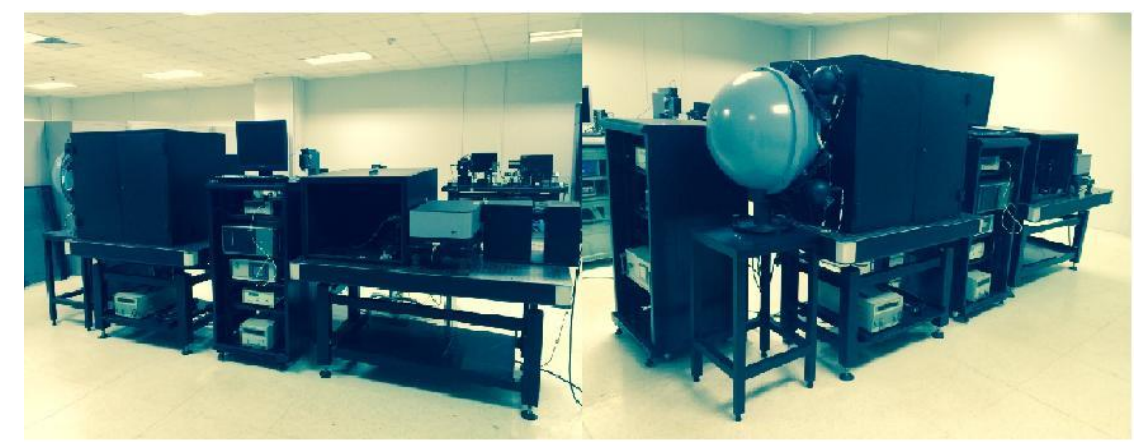

Figure 8. Experimental Facilities 
A sample of CMOS sensor was tested under constant temperature of $25^{\circ} \mathrm{C}$, with the removal of dark signals by black level correction. Images were collected from dark frame to irradiance saturation. For each irradiance level, 1000 frames of images were collected, and the average was calculated. The video response curve is plotted as shown in Figure 9, which indicates an obvious non-linearity.

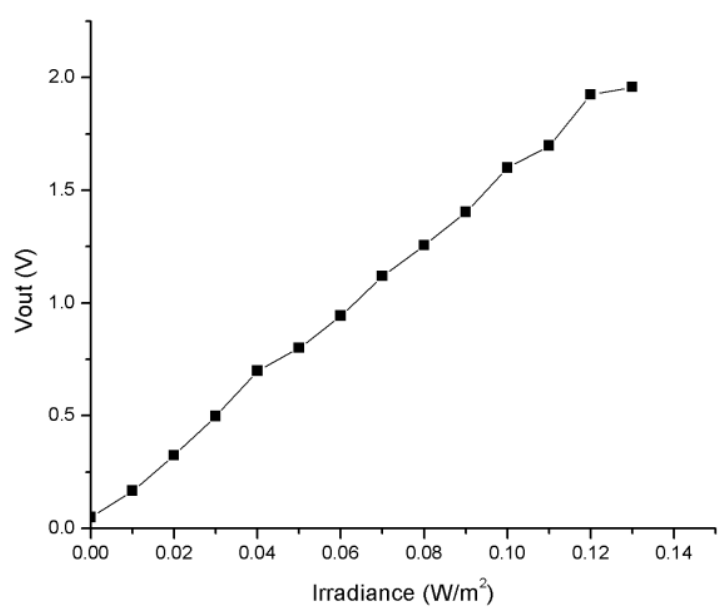

Figure 9. Measured Video Response Curve of CMOS Sensor

PRNU noise curve was further tested, and the test results are shown in Figure 10. It can be seen that PRNU noise factor decreases with the increase of irradiance. Therefore serious V/Q non-linearity exists in the sensor. MTF testing was carried out under Nyquist frequency, and the results are shown in Table 1. As the irradiance increases, MTF decreases considerably.

To locate the V/Q non-linearity, the non-linear interval of the video response curve was corrected by look-up table method. Then the video response curve and PRNU noise curve were tested again. The tests showed that the video response curve has an improved linearity after correction, with maximum non-linearity controlled under 5\%. PRNU noise curve is basically a horizontal straight line, as shown in Figure 11. The result of MTF testing under Nyquist frequency is shown in Table 1. Apparently, the test value of MTF increases after V/Q non-linearity is removed, and MTF generally stays constant regardless of the change of irradiance.

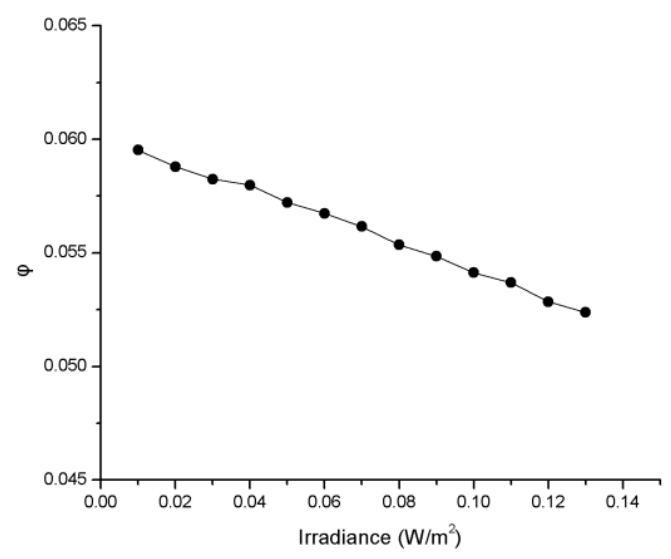

Figure 10. Measured PRNU Noise Curve of CMOS Sensor 


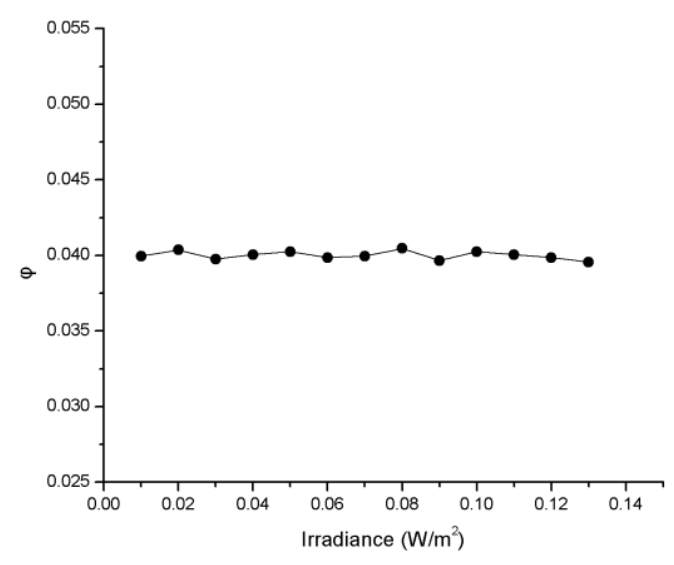

Figure 11. PRNU Noise Curve after Correction

Table 1. Results of MTF Testing

\begin{tabular}{c|cc}
\hline $\begin{array}{c}\text { Input } \\
\text { irradiance } \\
\left(\mathrm{W} / \mathrm{m}^{2}\right)\end{array}$ & $\begin{array}{c}\text { Result of MTF } \\
\text { testing under V/Q } \\
\text { non-linearity }\end{array}$ & $\begin{array}{c}\text { Result of MTF } \\
\text { testing under } \\
\text { linear conditions }\end{array}$ \\
\hline 0.0643 & 0.493 & 0.513 \\
0.0776 & 0.488 & 0.508 \\
0.0883 & 0.483 & 0.511 \\
0.0985 & 0.478 & 0.509 \\
\hline
\end{tabular}

The above experiments indicate that the proposed method can effectively locate V/Q non-linearity, thus providing the tool for design optimization and verification of correction effect.

\section{Conclusion}

We build the mathematical model of V/Q non-linearity for CMOS sensor and carry out a simulation analysis. The method of combining video response curve with PRNU noise curve for judging V/Q non-linearity is proposed. Experiments show that V/Q nonlinearity does have an impact on MTF, which is consistent with the simulation. The proposed method is capable of locating V/Q non-linearity in CMOS sensor.

\section{References}

[1] G. Gramegna, P. O'Connor, P. Rehak, S. Hart, "CMOS preamplifier for low-capacitance detectors. Nuclear Instruments and Methods in Physics Research A", 390, (1997), pp. 241-250.

[2] B Ben Bakir, S Menezo, J-M Fedeli, D J Moss, "Amorphous Silicon Nanowires with Low TwoPhoton Absorption, High Nonlinerity and Good Stability. ResearchGate, 5, (2014).

[3] S. Zhang, W. Han, L. Cui, "Research on Evaluation Method of CMOS Camara", 7th International Symposium on Advanced Optical Manufacturing and Testing Technologies, Proc. of SPIE Vol. 9282, 928238.

[4] R. Dai, Y. Zheng, H. Zhu, W. Kong, S. Zou, "A High Gain and High Linerity Current-Reused CMOS LNA Using Modified Derivative Superposition Technique With Bulk-Bias Control”, Microwave and Optical Technology Letters. vol. 56, no. 10, (2014).

[5] W. Jun, Y. Huiling, L. Yaxia, "Correction to The Nonuniformity of Multiple CCDs Butting Camera", Semiconductor Optoelectronics, vol. 6, no. 3, (2005), pp. 261-263.

[6] X. Jihong, H. Pu, L. Jun, "Non-Uniformity Correction of Large Area Array Color CCD Aerial Mapping Cameral", ACTA OPTICA SINICA, vol. 33, no. 7, (2013):0711003.

[7] C. Wansheng, Z. Jie, C. Hehao, "Correction method for pixel response nonuniformity of CCD", Opt. Precision Eng, vol. 16, no. 2, (2008), pp. 314-318. 
[8] D. J. Moss, R. Morandotti, A. L. Gaeta, M. Lipson, "New CMOS-compatible Platforms Based on Silicon Nitride and Hydex for Nonlinear Optics", Nature Photonics, vol. 7, (2013), pp. 597-607.

[9] J. F. Gunaman, T. Dosluoglu, "Scientific CMOS pixels. Conf. Proc. on Scientific Detectors for Astronomy", 300(2004), pp. 3-114.

[10] O. Yadid-Pecht, R. Etienne-Cummings. "CMOS Imagers: From Phototrans duction to Image Processing ", Kluwer Academic Publishers, (2004)

[11] Y. Shang, Y. Guan, W. Zhang, "A high dynamic range complementary metal-oxide-semiconductor (CMOS) camera using multi-slope response and an image reconstruction algorithm", Meas. Sci. Technol,(2009).

[12] M. R. Stanford, B. J Hadwen, "The noise performance of electron multiplying charge coupled devices", IEEE Trans. Electron. Devices, (2003).

[13] Brent McCleary, Antonio Ortega. Photo-response non-uniformity error tolerance testing methodology for CMOS imager systems. SPIE-IS\&T, 7242 (2009): 724216-1 724216-12.

[14] S. Yuanyuan, Z. Weigong, "Research on Evaluation Method of CMOS Imager", College of Information Engineering, (2010).

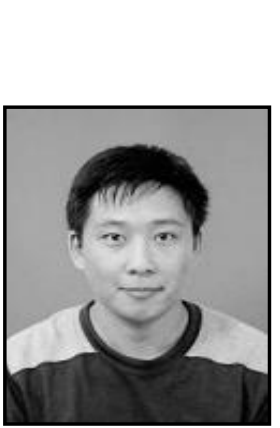

\section{Authors}

Jun-Lin Li. Jun-Lin Li, was born in Anshan, China, in 1984. He received the B.S. degree from Beihang University, Beijing, China in 2007, and the M.S. degree from Tianjin University, Tianjin, China in 2009. He is currently working toward the Ph.D. degree in University of Chinese Academy of Sciences, Beijing, China. His research area includes design and test technology of the space camera.

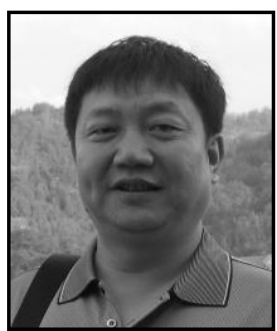

Yong-Fei Guo. Yong-Fei Guo, was born in Changchun, China, in 1961. He is currently a Professor and Ph.D. supervisor in University of Chinese Academy of Sciences, China. His research interests are photoelectric sensor information and remote sensing.

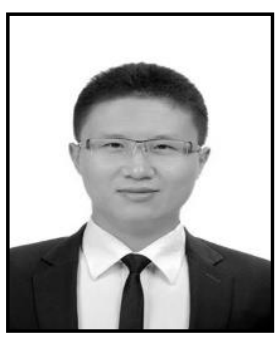

Taiji-Ji Lan. Tai-Ji Lan was born in Yushu, China, in 1988. He received the B.S. degree from Tianjin University, Tianjin, China in 2011, and the M.S. degree from Tianjin University, Tianjin, China in 2014. He is currently working toward the Ph.D. degree in University of Chinese Academy of Sciences, Beijing, China. His recent interests focus on testing technology of the space camera. 